\title{
Ambulance 3G
}

\author{
Konstantinos A. Banitsas ${ }^{1}$, Konstantinos Perakis ${ }^{2}$, Sapal Tachakra ${ }^{3}$, Dimitrios Koutsouris ${ }^{2}$, Georgios Konis ${ }^{2}$ \\ ${ }^{1}$ E\&CE, School of Engineering and Design, Brunel University, West London, England \\ ${ }^{2}$ Biomedical Engineering lab, National Technical University of Athens, Greece \\ ${ }^{3}$ A\&E department, North West London Hospitals, England
}

\begin{abstract}
Minimising the time required for a patient to receive primary care has always been the concern of the Accidents and Emergency units. Ambulances are usually the first to arrive on the scene and to administer first aid. However, as the time that it takes to transfer the patient to the hospital increases, so does the fatality rate.

In this paper, a mobile teleconsultation system is presented, based primarily on third generation mobile links and on Wi-Fi hotspots around a city. This system can be installed inside an ambulance and will permit high-resolution videoconferencing between the moving vehicle and a doctor or a consultant within a base station (usually a hospital). In addition to video and voice, high quality still images and screenshots from medical equipment can also be sent.

The test was carried out in Athens, Greece where a 3G system was recently deployed by Vodafone. The results show that the system can perform satisfactory in most conditions and can effectively increase the patient's quality of service, while having a modest cost.
\end{abstract}

Keywords_Ambulance, 3G, wireless, telemedicine

\section{INTRODUCTION}

One of the crucial factors when trying to improve the healthcare delivery in emergency situations is the minimisation of time required to provide primary care (and most importantly consultation) to the patient. In most cases an ambulance has to respond to an emergency call, administer first aid and in many cases carry the patient to an Accident and Emergency ward in a close by hospital. In Greece there are an average of 461000 ambulance calls every year. In the general area of Athens alone, an average of 730 ambulance calls take place each day with $27 \%$ of those patients in need to rush into an A\&E department (category A responses). The average time it takes for an ambulance to reach its destination in the city of Athens is 17 minutes [1].

Patients transferred that are in critical condition, would benefit by a faster access to medical expertise (that cannot be provided by staff operating the ambulance) and more specifically, direct interaction with either a doctor or a consultant.

One of the attempts that tried to improve the above situation and provide a wireless channel of information between the moving ambulance and a base station, was a project developed by the National Technical University of Athens in 1998; Emergency 112. The objective was to reduce treatment times, improve medical diagnosis, and minimise costs by developing an integrated portable medical device for Emergency Telemedicine. The transmission of critical biosignals (ECG, BP, HR, SpO2, Temperature) and images to an Emergency call centre enabled physicians to direct pre-hospital care more effectively [2]. However, as the system was using a second generation mobile telephony link $(2 \mathrm{G})$ it was limited to a speed of $9.6 \mathrm{Kbps}$. Having just this bandwidth available, only low resolution static images and text-like patient data could be sent over the air.

As the situation in mobile telephony improved General Packet Radio Service (GPRS) came into use (2.5G) and allowed for a bandwidth of about $50 \mathrm{Kbps}$, permitting users to send static images of modest quality [3].

The deployment of the third generation of mobile telephony $(3 \mathrm{G})$ has created many new possibilities for overthe-air communication and more specifically for distant health care delivery. $3 \mathrm{G}$ systems can support higher data rates thus allowing for a range of new applications [4].

$3 \mathrm{G}$ systems utilise the Universal Mobile Telecommunication Services (UMTS) technology. When conventional $2 \mathrm{G}$ mobile terminals move from one cell to another, they have to disassociate from the old cell and associate to a new cell. This process of "handover" (HO) introduces small gaps in communication that can severely affect time sensitive processes like videoconferencing. In contrast with 2G, UMTS uses "soft hand-over" where a mobile terminal switches from one cell to another in such a way that there is a point in time that it communicates with both cells. That translates to a more stable flow of data, essential in real time applications [4].

As the number of $3 \mathrm{G}$ cells is lower than those supporting $2.5 \mathrm{G}$ when a mobile terminal gets out of the $3 \mathrm{G}$ coverage area it will have to fallback into GPRS speed, until it regains a $3 \mathrm{G}$ signal. The whole operation of disassociating with $3 \mathrm{G}$ and registering in a $2.5 \mathrm{G}$ network introduces considerable delays in communication and data transfer.

Alternatively, as there are an increasing number of access points supporting WLANs (usually close to highly populated areas like city centres, airports, etc), they can be used by a system such as the one described in this paper and can offer faster connection at a lower cost, compared to the $3 \mathrm{G}$.

In the remaining of this article there will be a presentation of a system that can be installed in a moving ambulance and by taking advantage of $3 \mathrm{G}$ communication can establish a video / audio / image link with a base hospital so doctors or consultants can offer their expert opinion to a patient in need, from the time this patient enters the ambulance.

\section{Methodology}

A series of 17 trial runs were conducted in the city of Athens. This city was chosen for two distinct reasons: First, 
the traffic within the centre of the city is quite high thus the average time needed for the ambulances to reach their destination is above average so, patients would benefit more by the deployment of such a telemedical system. Secondly, 3G mobile telephony was recently deployed by Vodafone thus presenting system developers with an advantage as the spectrum had a very low utilisation rate.

In the trial runs an ambulance was equipped with the following [Fig. 1]:

- A high end, light, laptop computer.

- A quality camcorder connected to the above laptop, that could focus and white balance under various lighting conditions. It could also support for high resolution still imaging.

- Either a PCMCIA card that supports $3 \mathrm{G}$ communication between a laptop and a $3 \mathrm{G}$ network or a $3 \mathrm{G}$ phone that acted as a modem (wired using a USB port or wireless using bluetooth).

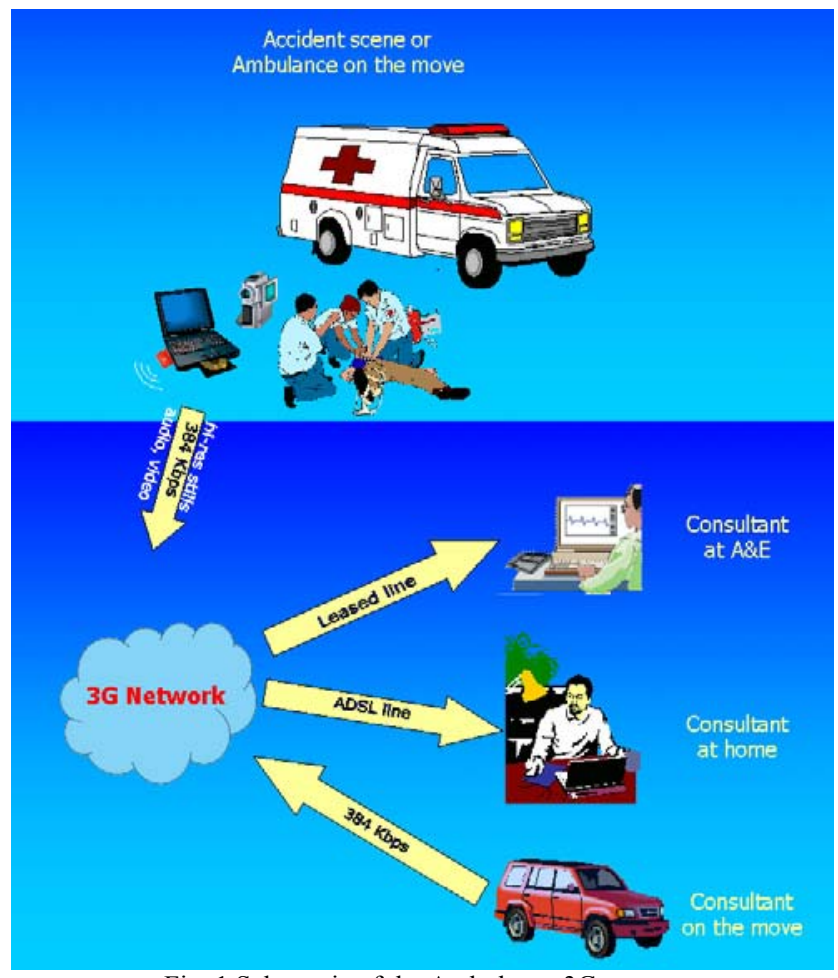

Fig. 1 Schematic of the Ambulance 3G system

Within the laptop a standard videoconferencing programme was running allowing for point-to-point communication to the base station. The base station resided in a hospital where either a doctor or a consultant could use a standard computer to establish a link with the moving ambulance [Fig. 1]. An average quality web camera was installed on the base computer and allowed for two-way videoconferencing. That was not an essential requirement of the test but improved the feeling of the users and especially the patients, as they tend to be calmer viewing the image of a doctor. It also allowed for the consultant to visually demonstrate a method to the treating personnel. However, two-way videoconferencing posed an extra strain to the communication channel and lowered the quality of the ambulance-to-base station link.

The routes followed by the ambulance, consisted by a mixture of UMTS and GPRS covered areas: Only a relatively low percentage of the geographical area of Athens is covered by $3 \mathrm{G}$ cells, centred close to the main routes and highways. For the remaining area the system had to fallback to GPRS thus considerably lowering the available speed. Several road speeds were tested, from a standstill position to a top speed of $140 \mathrm{Km} / \mathrm{h}$. Finally, alternative routes were followed, that included four road tunnels, three times travelling sideways to a mountain and several urban canyons.

Various scenarios were run mostly in relation with videoconferencing and included the actual trauma, real-time ECG signals, supervision of the actions of the ambulance personnel, etc. Also, a number of different still images were transmitted that included patient and trauma details, paper printed information, screenshots of medical equipment and other patient details.

As there had to be some kind of network performance indicator, on the mobile side of the system, two network monitoring programmes were running continuously: one was provided with the PCMCIA card and indicated the network connection (UMTS or 3G) and the speed connected, while the other displayed information on channel utilisation, packet size, protocol usage and specific speed on every instant.

\section{RESULTS}

Regardless of some specific problems, the overall system performance has met or even exceeded the expectations of both the engineers and the medical personnel and showed that the system can be effectively deployed on a larger scale.

\section{A. Engineering}

The videoconferencing sessions produced relatively clear video in general. The algorithm used with most videoconferencing programmes is H.263 (a simplified version of MPEG-4 algorithm) [5]. Fortunately the bandwidth was high enough for a satisfactory video of 10-15 frames per second (fps) and a choice of resolution between 160x 120, 320x240 and 640x480 pixels. Windows embedded videoconferencing software was proven to be sufficient for the task. Apart from audio and video, it also supported file transfer (FTP) including images and patient data.

The initial speed of connection reported by the PCMCIA utility was usually $384 \mathrm{Kbps}$, reflecting a $3 \mathrm{G}$ coverage area. The speed was stable with very little margin of fluctuation. When connected to a $2.5 \mathrm{G}$ cell, the speed dropped to about $40 \mathrm{Kbps}$ but with relatively heavy fluctuations. As the system handed over from one $3 \mathrm{G}$ cell to 
another, the transaction was very smooth and literally undetectable to the user, as the mobile terminal was connected to both cells during the HO period.

When the system handed-over from a $3 \mathrm{G}$ cell into a $2.5 \mathrm{G}$, there were some inevitable delays as the terminal had to reregister to another cell. Delays varied from 5-15 seconds and there was an instance where communication was lost for more than one minute and the whole session had to be re-established. For a total period of 23 hours and an area of about 180 square kilometres, there were 9 recorded instances where the communication fell back from $3 \mathrm{G}$ to 2.G and for a total of 17 minutes.

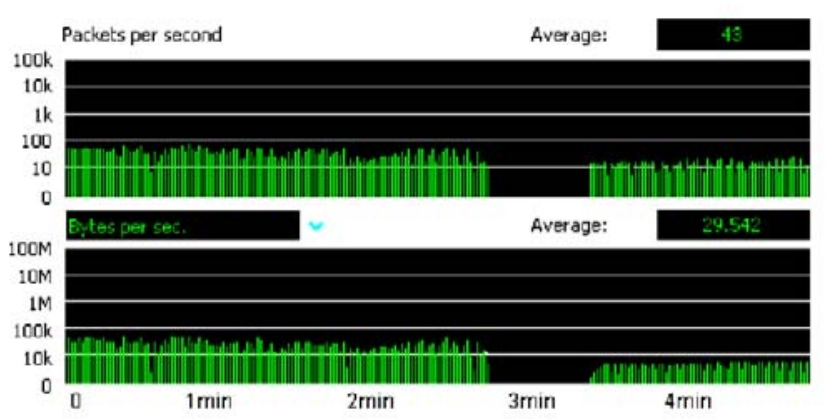

Fig. 2. Network reported throughput: left section indicates $3 \mathrm{G}$, right section is the fallback into GPRS, the gap in the middle is the hand-over

In the case that there was no emergency need for the patient to be transferred to an A\&E ward, a different approach could be followed: the same laptop computer can also accept a WLAN card (IEEE 802.11a, b or g) making it capable of connecting to the hospital site, via a Wi-Fi hotspot. This increases the speed of communication dramatically (in the case of IEEE $802.11 \mathrm{~b}$ to a useful data rate of 2.5-3.5 Mbps) and allows for lower compression rates resulting to a clearer videoconferencing session.

This approach was tested as an alternative to the $3 \mathrm{G}$ one, by placing access points in specified locations around a test site. The results were encouraging while the behaviour of the system resembled that of indoors teleconsultation systems [6]. The disadvantage of this option is that the ambulance and staff should be on a relatively stationary position to maintain connection to the access point.

\section{B. Telemedical}

During the research of this system and for a period of 7 days, a number of doctors, patients and other personnel were involved:

- A total of 17 patients were transferred using an ambulance that the above system was installed into.

- Four consultants were asked to evaluate the results sent by the system (video, image and sound) and make a prognosis on the patient, based on this data.

- Two engineers continuously researched into the communicational properties of the system.

All the patient cases researched, produced acceptable results for the remote consultants both for videoconferencing and for still imaging. The overall quality of the outputs (based on questionnaires given to the remote doctors) was: poor $4.2 \%$, acceptable $25.4 \%$ and good $70.2 \%$ [Table 1].

TABLE 1

SUMMARY OF DOCTORS' FEEDBACK REGARDING THE AUDIO / VIDEO QUALITY OF THE SYSTEM

\begin{tabular}{lccc}
\hline & Poor $\%$ & Acceptable $\%$ & Good $\%$ \\
\hline Image clarity & & 35 & 65 \\
Colour & & 20 & 80 \\
Depth versatility & & 8 & 92 \\
Sound & 10 & 45 & 55 \\
Audio / Video delay & 15 & 15 & 75 \\
Total delay & 5 & 35 & 50 \\
X-ray detail & 20 & 75 \\
\hline
\end{tabular}

Sound was also acceptable although the relatively high background noise created by the ambulance moving (especially with the use of siren) lowered the quality of videoconferencing.

Transmitting a still image was a straightforward task. The same high quality camcorder that was used for videoconferencing, could also be used as a digital camera so still images of either 1 or 2 Mpixels could be transmitted to the laptop computer and from then to the $3 \mathrm{G}$ network. A still image of 1Mpixel took about 10-15 seconds to be transmitted while a $2 \mathrm{Mpixel}$ one required twice as much time. The quality of the images was very good, even under poor lighting conditions such as fluorescent lighting inside the ambulance. The camera could focus from actual zero to infinity making this ideal for pointing at a patient's trauma or any other body details. Even pointing the camera straight to various medical equipment within the ambulance, produced a very clear and sharp image. That included both TFT and CRT screens and monitors. Although there was little need of that, the most difficult task was proven to be the transmission of films (x-rays, CT, MRI, etc) [6].

Finally, the system could also act as an on-line terminal to the hospital's network, thus allowing the access of information in the hospital's database (patient's history, drug prescription, etc).

\section{DisCUSSION}

In engineering terms, the system had an acceptable videoconferencing quality when the ambulance was within a $3 \mathrm{G}$ coverage area. Moving into a GPRS area posed a problem, as the bandwidth was not enough to accommodate the load. Sound and still imaging was very satisfactory even in GPRS conditions rendering the system useful, even in areas that do not have support for $3 \mathrm{G}$ communication.

However, it is more than certain that the number of $3 \mathrm{G}$ cells within every European country will keep increasing much like $2 \mathrm{G}$ cells did in the past. For that reason, research like the one presented in this article is bound to have an increasingly high field of application and a guaranteed success as more and more areas are covered by UMTS. 
The cost of the additional hardware needed to be installed in an ambulance, does not exceed 6000 Euros (about 4000 pounds). Airtime needed for the videoconferencing session is charged by the provider. Most test session lasted from 3-10 minutes transmitting 3-15 MB of data. It is estimated that the average cost for the airtime of each session would be about 5-20 Euros (3-13 pounds). The overall cost of the system (installation and running cost) is very low and should make the system desirable for full deployment.

As this is a telemedical application, the security of the system is always of high interest, as sensitive medical data are to be transmitted over the air. A Virtual Private Network (VPN) was used to create a secure tunnel from source to destination. Fortunately, the software that came along with the PCMCIA $3 \mathrm{G}$ card included such a security wizard that allowed creating such a secure connection. The data overhead of the VPN solution was minimum and remained unnoticeable to the end users [6,7].

At this point it is important to mention that this system can be installed to work the other way around: from a base station in the hospital (possibly A\&E ward) to a mobile station where a consultant may be. It is clear that consultants have to be reachable as much as possible. The above system can be installed in a mobile vehicle (the consultant's car for example) and allow for communication between the car and the A\&E ward. [8]

\section{CONCLUSION}

The general opinion formed by the doctors was that this system produced solid results that could be used for improving the quality of service to the patients. More specifically, all prognoses made through using the system, agreed with the final diagnoses of the patients; even though further tests had to be made to justify each diagnosis.

The general idea is that using this system, small groups of consultants can be housed on one hospital and serve a wide area within a city, or an entire city itself.

Overall, it is clear that a high quality, low cost system like this can effectively assist on minimising the time a patient needs to get primary consultation and effectively save lives.

\section{ACKNOWLEDGMENT}

All parties involved in this research, would like to thank Vodafone Greece for their support, both for the hardware and the airtime donated.

\section{REFERENCES}

[1] Greek Ministry of Health, National Centre of Emergency Response, Annual Report - 1998, Athens, Aug 1999

[2] Pavlopoulos S., Kyriakou E., Berler A., Koutsouris D. "Emergency telemedicine applications using mobile and internet communication links - The AMBULANCE Project", Proceedings of EURO-MED NET 98 Conference, Nicosia, Cyprus, pp. 281-282, 1998

[3] Konstantinos A. Banitsas, P. Georgiadis, Sapal Tachakra, D. Kavouras, "Using handheld devices for real-time wireless Teleconsultation", IEEE EMBC 2004 San Francisco, pp. 3105-3108, Sep 2004

[4] Jonathan P. Castro, "The UMTS Network and Radio Access Technology Air Interface Techniques for Future Mobile Systems", John Wiley \& Sons Ltd, England, 2001

[5] lain E. G. Richardson, "Video Codec Design Developing Image and Video Compression Systems", John Wiley \& Sons Ltd, England, 2002

[6] Konstantinos Banitsas, "MedLAN: Compact Mobile Computing System for Wireless Information Access in Emergency Hospital Wards", $\mathrm{PhD}$ thesis, Brunel University, London, May 2004

[7] Thomas J. Owens, Sapal Tachakra, Konstantinos A. Banitsas, Robert S. H. Istepanian, "Securing a Medical Wireless LAN System", IEEE EMBC conference, Istanbul, vol. 4, pp. 3552-3555, Oct 2001

[8] Konstantinos A. Banitsas, Sapal Tachakra, R.S.H. Istepanian, "Operational Parameters of a Medical Wireless LAN: Security, Range and Interference issues", IEEE EMBC conference, Houston, vol. 3, pp. 1889-1890, Oct 2002 